\title{
From Tweeds to Tweets: \\ Democratizing and Diversifying the \\ Ivory Tower and Science \\ Communication through Social \\ Media
}

JENNIFER GLASS

\begin{abstract}
The advent of social media platforms has revolutionized science education and communication. For the first time in human history, anyone with internet access can communicate directly to each other, regardless of education or location. This poses a significant opportunity for scientists to reach the world with their findings, and to show the world how science is accessible, inspirational, and essential. In this talk, I will highlight a few lessons from my journey of being increasingly outspoken on public social media platforms about scientific discoveries and the contributions of previously "hidden figures" in science.
\end{abstract}

